\title{
Is the Kluyver effect in yeasts caused by product inhibition?
}

\author{
Ruud A. Weusthuis, Marijke A. H. Luttik, W. Alexander Scheffers, \\ Johannes P. van Dijken and Jack T. Pronk
}

Author for correspondence: Jack T. Pronk. Tel: +31 15 782387. Fax: +31 15782355.

Department of Microbiology and Enzymology, Kluyver Laboratory of Biotechnology, Delft University of Technology, Julianalaan 67, 2628 BC Delft, The Netherlands
Candida utilis CBS 621 exhibits the Kluyver effect for maltose, i.e. this yeast can respire maltose and is able to ferment glucose, but is unable to ferment maltose. When glucose was pulsed to a maltose-grown, oxygen-limited chemostat culture of $\boldsymbol{C}$. utilis, ethanol formation from glucose started almost instantaneously, indicating that the enzymes needed for alcoholic fermentation are expressed in maltose-grown cells. However, the addition of glucose inhibited maltose metabolism. To eliminate a possible catabolite inhibition and/or repression of enzyme activities involved in maltose metabolism, the effect of simultaneously feeding glucose and maltose to an oxygen-limited, maltose-grown chemostat culture was studied. In this case, the glucose concentration in the culture remained below $0.1 \mathrm{mM}$, which makes glucose catabolite repression unlikely. Nevertheless, maltose metabolism appeared to cease when the culture was switched to the mixed feed. Based on the outcome of the mixed-substrate studies, it was postulated that the Kluyver effect may be caused by feedback inhibition of maltose utilization by ethanol, the product of fermentative maltose metabolism. If ethanol suppresses the utilization of non-fermentable disaccharides, this would provide a phenomenological explanation for the occurrence of the Kluyver effect: accumulation would then not occur and the rate of maltose metabolism would be tuned to the culture's respiratory capacity. This hypothesis was tested by studying growth of C. utilis CBS 621 and Debaryomyces castellii CBS 2923 in aerobic batch cultures on mixtures of sugars and ethanol. With both yeasts diauxic growth was indeed observed on mixtures of ethanol and a disaccharide that gives rise to the Kluyver effect, with ethanol being the preferred substrate. In contrast, sugars which could be fermented were either utilized simultaneously with ethanol or preferred over this substrate.

Keywords: Kluyver effect, yeasts, fermentation, disaccharide metabolism, product inhibition

\section{INTRODUCTION}

Many facultatively fermentative yeast species show a peculiar behaviour with respect to the utilization of certain disaccharides. Depending on the yeast species, some disaccharides cannot be fermented, although respiration of the disaccharides and fermentation of the component hexose(s) are both possible (Weusthuis $e t$ al., 1994). This physiological phenomenon is called the Kluyver effect (Kluyver \& Custers, 1940; Sims \& Barnett, 1978). Of the 215 glucose-fermenting yeast species 96 exhibit the Kluyver effect for at least one disaccharide (Barnett et al., 1990). Table 1 shows the widespread occurrence of the Kluyver effect among the facultatively fermentative yeasts that are presently known.

Although the mechanism responsible for the Kluyver effect has not yet been elucidated, it must somehow be related to differences in the metabolism of disaccharides and monosaccharides. Target reactions at which control of disaccharide metabolism may be exerted are sugar uptake and/or disaccharide hydrolysis. Also, the possibility that specific kinases are involved in transport-associated phosphorylation of hexoses and phosphorylation of cytosolic hexose molecules generated from disaccharide 
Table 1. Screening of facultatively fermentative yeast species for their ability to ferment a number of disaccharides and for the occurrence of the Kluyver effect

Yeasts included in the screening could grow on the disaccharide listed and were able to ferment glucose. Data were obtained from a taxonomic handbook (Barnett et al., 1990). Only yeast species of which all strains showed positive results are listed.

\begin{tabular}{|lcc|}
\hline \multirow{2}{*}{ Sugar } & \multicolumn{2}{c|}{$\begin{array}{c}\text { No. of yeast species that } \\
\text { show: }\end{array}$} \\
\cline { 2 - 3 } & Fermentation & $\begin{array}{c}\text { Kluyver } \\
\text { effect }\end{array}$ \\
\hline Maltose & & \\
Methyl- $\alpha$-D-glucopyranoside & 15 & 41 \\
Sucrose & 7 & 45 \\
Trehalose & 64 & 32 \\
Melibiose & 29 & 35 \\
Lactose & 6 & 1 \\
Cellobiose & 1 & 11 \\
Melezitose & 7 & 43 \\
Raffinose & 1 & 55 \\
\hline
\end{tabular}

hydrolysis (Clifton et al., 1993) cannot be neglected as a possible cause of the Kluyver effect.

In oxygen-limited chemostat cultures of Candida utilis grown on maltose, the amount of maltose that can be metabolized is limited by the amount of oxygen available for respiration. When the oxygen feed to these cultures is decreased, maltose is only partially consumed and alcoholic fermentation is not observed (Weusthuis et al., 1994). In contrast, glucose-limited cultures of C. utilis grown at low oxygen feeds simultaneously respire and ferment glucose. These experiments indicate that in $C$. utilis, oxygen availability is not a key factor in the Kluyver effect for maltose. Instead, the Kluyver effect appears to reflect an intrinsic inability to ferment particular disaccharides (Weusthuis et al., 1994).

Regardless of the molecular mechanism, the observed inability of Kluyver-positive yeasts to form ethanol from disaccharides may be caused in two distinct ways: either the presence of disaccharides directly or indirectly inhibits the occurrence of alcoholic fermentation or, alternatively, extracellular free monosaccharides are required to induce alcoholic fermentation. The primary aim of the present work was to discriminate between the above two possibilities by studying the metabolism of mixtures of glucose and maltose by $C$. utilis. To avoid repression of maltosemetabolizing enzymes, glucose-limited chemostat cultures were used, in which the low residual glucose concentrations prevent such phenomena.

Based on the experimental results, a hypothesis was formulated which gives a phenomenological explanation of the Kluyver effect. This hypothesis was verified by studying the growth of the Kluyver-positive yeasts
Candida utilis and Debaryomyces castellii on mixtures of various sugars and ethanol.

\section{METHODS}

Organisms and maintenance. C. utilis CBS 621 and D. castellii CBS 2923 were obtained from the Centraalbureau voor Schimmelcultures (Delft, The Netherlands) and maintained on malt agar slants at $4{ }^{\circ} \mathrm{C}$.

Chemostat cultivation. Chemostat cultivation was performed in 21 fermenters (Applikon) at a dilution rate of $0 \cdot 10 \mathrm{~h}^{-1}$, a temperature of $30^{\circ} \mathrm{C}$ and a stirrer speed of 750 r.p.m. The culture $\mathrm{pH}$ was maintained at $5 \cdot 0$ by automatic addition of $2 \mathrm{M}$ $\mathrm{KOH}$, controlled by an Applikon ADI-1020 biocontroller. To avoid loss of volatile metabolites, the condenser was cooled to $2{ }^{\circ} \mathrm{C}$, using a cryostat. The working volume of the culture was kept at 1.01 by removal of effluent from below the surface of the culture, controlled by an Applikon electrical level controller. This set-up ensured that biomass concentrations in the effluent line differed by less than $1 \%$ from those in samples taken directly from the culture. Samples for biomass, substrate and product analysis were taken from the effluent line. The mineral medium, supplemented with vitamins and trace elements, was prepared as described by van Leeuwen et al. (1992). Glucose or maltose were added to the media after separate sterilization (Weusthuis et al., 1993). The purity of the chemostat cultures was routinely checked by phase-contrast microscopy at $1000 \times$ magnification.

Oxygenation of the chemostat cultures. Oxygen was added to the cultures as air $\left(60 \mathrm{ml} \mathrm{min}^{-1}\right)$, with a constant temperature of $20^{\circ} \mathrm{C}$, using a Masterflex peristaltic pump. In the oxygenlimited cultures, the overall gas-flow rate into the cultures was maintained at $560 \mathrm{ml} \mathrm{min}^{-1}$ by supplementary addition of nitrogen gas ( $500 \mathrm{ml} \mathrm{min}^{-1}$ ) via a mass-flow controller (Brooks). Addition of nitrogen gas ensured good mixing of the air with the culture fluid. To minimize diffusion of atmospheric oxygen into the oxygen-limited cultures, the entire fermentation set-up (including medium reservoir and effluent vessel) was equipped with Norprene tubing (Cole Parmer Inc.). The dissolvedoxygen concentration in the cultures was monitored with a polarographic oxygen electrode (Ingold).

Batch cultivation. Yeasts were pregrown in $100 \mathrm{ml}$ shake flasks on $20 \mathrm{ml}$ of the mineral medium described above, with an initial $\mathrm{pH}$ of 6.0 and $5.0 \mathrm{~g}$ glucose $\mathrm{l}^{-1}$. Batch cultivation took place in the fermenters described above with an initial working volume of $1.5 \mathrm{l}$. The $\mathrm{pH}$ was controlled between 4.9 and 5.1 by automatic addition of $2 \mathrm{M} \mathrm{KOH}$ or $1 \mathrm{M} \mathrm{H}_{2} \mathrm{SO}_{4}$. The stirrer speed was kept between 750 and 1250 r.p.m., the air-flow rate into the culture was $1.01 \mathrm{~min}^{-1}$. The dissolved-oxygen tension in the culture was kept above $50 \%$ air saturation by manual adjustment of the stirrer speed. The temperature was $30^{\circ} \mathrm{C}$ for cultures of $C$. utilis or $25^{\circ} \mathrm{C}$ for cultures of $D$. castellii.

Metabolite analysis. Glucose and maltose concentrations were determined as described by Weusthuis $e$ t al. (1993). The lactose concentration was measured with the same assay as maltose, using $\beta$-galactosidase instead of $\alpha$-glucosidase. Acetate was determined by HPLC (Weusthuis et al., 1993). Ethanol concentrations were determined with a colorimetric assay kit (EK 001, Leeds Biochemicals). The maximal standard deviation for glucose and lactose was $0 \cdot 2 \mathrm{~g} \mathrm{l}^{-1}$, for ethanol $0 \cdot 1 \mathrm{~g} \mathrm{l}^{-1}$, for maltose $0.2 \mathrm{~g} \mathrm{l}^{-1}$ in the absence of glucose and $0.8 \mathrm{~g} \mathrm{l}^{-1}$ in the presence of glucose.

Biomass determinations. Dry weights of culture samples were determined using a microwave oven and $0.45 \mu \mathrm{m}$ membrane filters as described by Postma et al. (1989). Parallel samples varied by less than $1 \%$. 


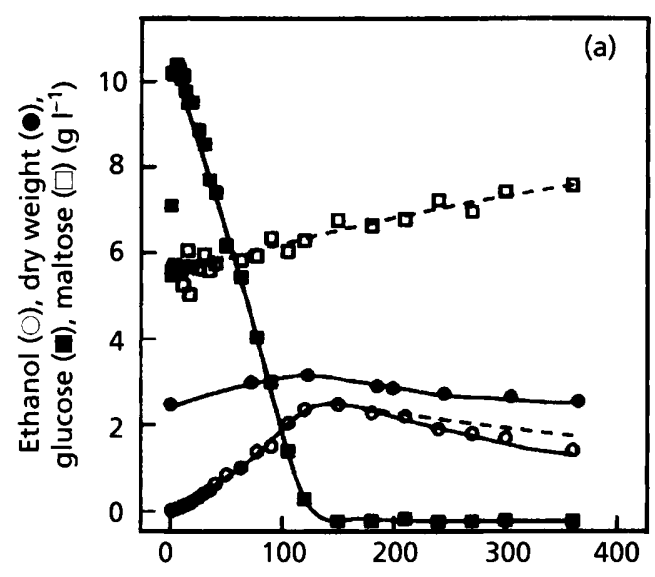

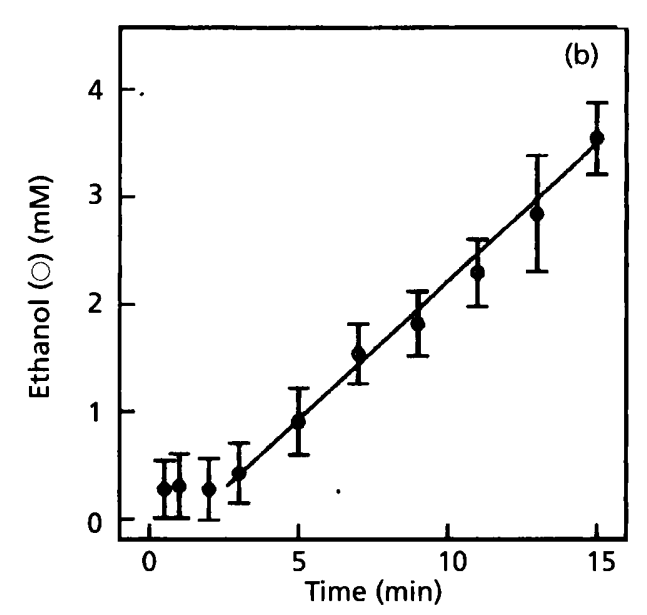

Fig. 1

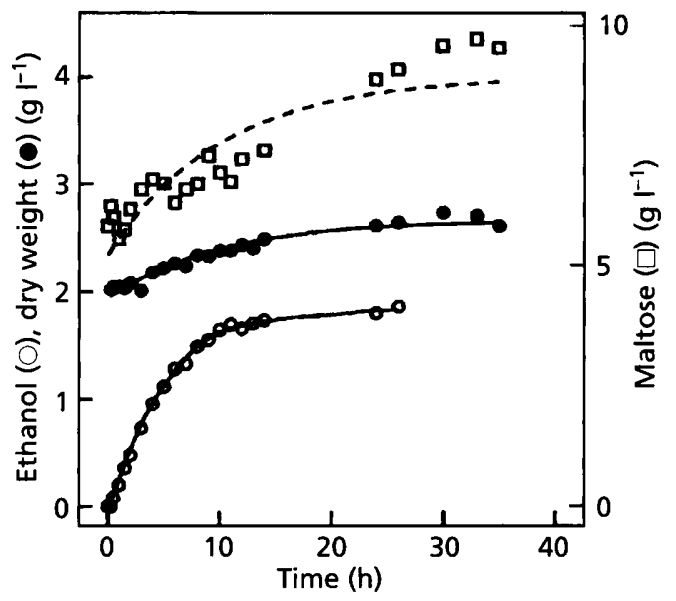

Fig. 2

Fig. 1. (a) Effect of the addition of glucose $\left(10 \mathrm{~g} \mathrm{l}^{-1}\right.$ at $\left.t=0\right)$ to an oxygen-limited chemostat culture of C. utilis CBS 621 with $10 \mathrm{~g}$ maltose monohydrate $\mathrm{I}^{-1}$ as carbon source $\left(D=0.1 \mathrm{~h}^{-1}\right)$ on biomass, maltose and ethanol concentration. The dashed lines indicate the expected profile in the case of wash-in kinetics (maltose) or wash-out kinetics (ethanol). (b) Ethanol production during the first minutes of the experiment.

Fig. 2. Effects of the addition of glucose $\left(10 \mathrm{gl}^{-1}\right.$ at $\left.t=0\right)$ to the reservoir medium of an oxygen-limited chemostat culture of $C$. utilis CBS 621 growing on $10 \mathrm{~g}$ maltose monohydrate $l^{-1}$ as carbon source $\left(D=0.1 \mathrm{~h}^{-1}\right)$. Ethanol was detected $0.5 \mathrm{~h}$ after the addition of glucose (data not shown).

Optical densities at $660 \mathrm{~nm}$ of culture samples were measured with a Vita Lab 20 spectrophotometer. When the optical density was above $0 \cdot 3$, the samples were diluted with demineralized water. Control experiments showed that this procedure assured a linear relationship between optical density and biomass dry weight. The standard deviation of the measurements was less than $2 \%$ of the measured value.

\section{RESULTS}

\section{Fermentative capacity of maltose-grown cells}

One of the proposed causes of the Kluyver effect is the absence, during growth on disaccharides, of one of the enzyme activities responsible for the conversion of glucose into ethanol. For example, Sims et al. (1991) and Sims \& Barnett (1991) proposed that the Kluyver effect could be caused by the absence of the enzymes pyruvate decarboxylase and/or alcohol dehydrogenase. However, in our studies it has been observed that during oxygen- limited growth of the Kluyver-positive yeast Candida utilis on maltose, these enzymes are present at high levels (Weusthuis et al., 1994). This indicates that in C. utilis the apparent inability to perform alcoholic fermentation of maltose is not due to the absence of these fermentative key enzymes. Nevertheless, the detection of these enzyme activities in cell-free extracts does not necessarily imply that they are also functional in vivo. To study whether maltose-grown $C$. utilis cells are capable of fermenting glucose, addition of glucose to oxygen-limited, maltosegrown cultures of this yeast was studied.

In a steady-state oxygen-limited culture of $C$. utilis grown on $10 \mathrm{~g}$ maltose $\mathrm{l}^{-1}$, alcoholic fermentation did not occur. Instead, the amount of maltose that could not be respired was not consumed, resulting in a residual maltose concentration of $4 \mathrm{~g} \mathrm{l}^{-1}$. When glucose $\left(10 \mathrm{~g} \mathrm{l}^{-1}\right)$ was added to this culture (Fig. 1a), ethanol formation could be detected in culture supernatants approximately $2 \mathrm{~min}$ 
after the pulse (Fig. 1b). The rate of ethanol formation was linear during the first $15 \mathrm{~min}$, confirming that all enzymes needed for the conversion of glucose into ethanol were present in the maltose-grown culture. The apparent 2 min delay before the onset of ethanol formation may be caused by the experimental procedures (sampling time, sensitivity of the ethanol assay). Also, the possibility of short-term activation/inactivation processes involving key enzymes of fermentative glucose metabolism cannot be excluded.

During the glucose-pulse experiment, both the addition of maltose-containing medium to the fermenter and the removal of culture effluent continued. Upon addition of glucose, an increase of the maltose concentration in the culture was observed (Fig. 1), suggesting that the presence of glucose interfered with maltose utilization. One of the possible causes of this interference is catabolite repression of maltose utilization by glucose, a phenomenon well documented in, e.g. Saccharomyces cerevisiae (Görts, 1969; Peinado \& Loureiro-Dias, 1986). In theory, glucose repression can be circumvented if a steady-state culture growing on maltose is switched to a medium feed containing both maltose and glucose. In this way, the glucose enters the culture slowly and can in principle be used immediately, thus avoiding accumulation of repressing glucose concentrations in the culture. Previous experiments with $S$. cerevisiae had shown that switching maltose-grown cultures to mixtures of maltose and glucose indeed did not result in glucose accumulation, but allowed simultaneous utilization of glucose and maltose. The residual glucose concentrations in these cultures were sufficiently low to prevent glucose catabolite repression of maltose-utilizing enzyme systems (Weusthuis et al., 1993).

\section{Chemostat cultivation of $C$. utilis on mixtures of glucose and maltose}

Addition of glucose $\left(10 \mathrm{~g} \mathrm{l}^{-1}\right)$ to the reservoir medium of an oxygen-limited, steady-state chemostat culture of $C$. utilis growing on $10 \mathrm{~g}$ maltose $\mathrm{l}^{-1}$ caused an increase of the residual maltose concentration in the culture. This increase followed wash-in kinetics until, eventually, the maltose concentration in the culture became equal to the concentration in the reservoir medium (Fig. 2). Apparently, simultaneous feeding of glucose and maltose caused a complete inhibition or suppression of maltose utilization.

During the transient-state experiment shown in Fig. 2, the glucose concentration in the culture remained below the detection limit of the glucose assay (approximately $0 \cdot 1 \mathrm{mM}$ ). Therefore, suppression of maltose utilization by glucose catabolite repression or inactivation seemed unlikely, although we could not exclude the possibility that even extremely low glucose concentrations inhibit maltose utilization in $C$. utilis. However, the rapid accumulation of ethanol after addition of glucose to oxygen-limited, maltose-grown cultures (Figs 1 and 2) indicates another explanation, namely an effect of ethanol or a related product of fermentative glucose metabolism

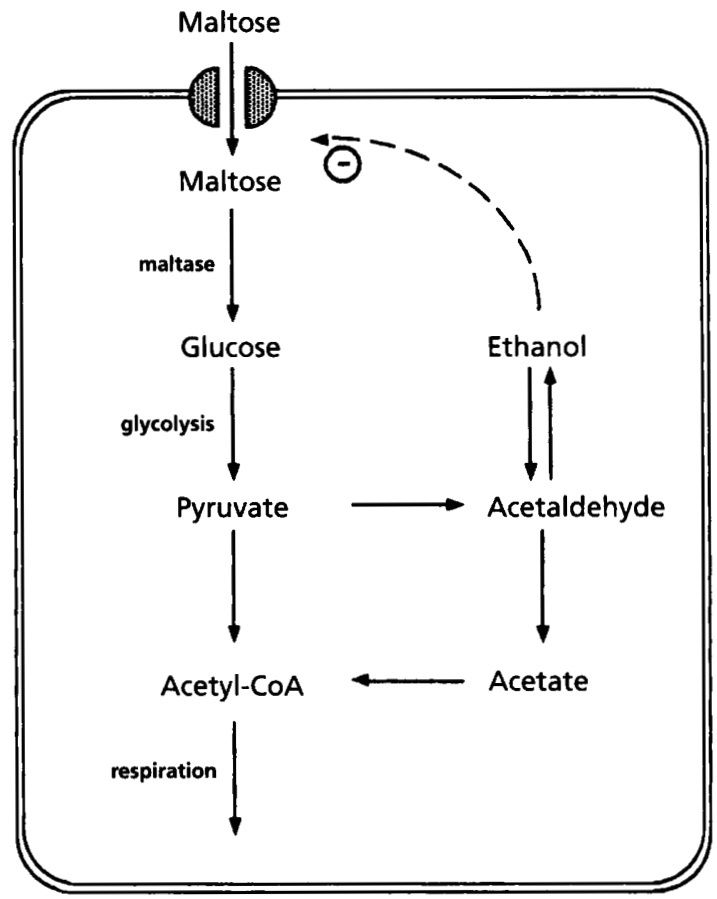

Fig. 3. Feedback inhibition of disaccharide utilization (for example via an effect on sugar transport) as a possible cause of the Kluyver effect in yeasts. Build-up of disaccharide fermentation products to a critical level results in inhibition of disaccharide uptake. Inhibition of disaccharide uptake can be relieved by the respiratory degradation of the inhibitory metabolite. This mechanism tunes the rate of disaccharide uptake to a value that is sufficiently low not to result in alcoholic fermentation.

on maltose metabolism. The products of fermentative metabolism of maltose and glucose are expected to be identical. Therefore, if fermentation products suppress or inhibit maltose metabolism, this should result in a feedback inhibition of maltose utilization once ethanol is formed from this substrate. This mechanism would offer a phenomenological explanation of the Kluyver effect that has so far not been mentioned in the literature: it would limit the rate of maltose utilization to the rate that can be accomplished without the occurrence of alcoholic fermentation (Fig. 3). The most likely target for this type of regulation would appear to be the disaccharide permease, since regulation of subsequent metabolic reactions could result in intracellular accumulation of disaccharides or other metabolites.

An indication that product inhibition may indeed be involved in the Kluyver effect is provided by the data shown in Fig. 1(a). After the complete consumption of the glucose that was pulsed into the culture, the concentration of ethanol decreased faster than wash-out kinetics predicts, indicating ethanol consumption by the culture. Maltose, however, was still not utilized. As long as ethanol was present in the culture, the maltose concentration continued to increase, according to wash-in kinetics, as a result of the feed of medium to the culture. 

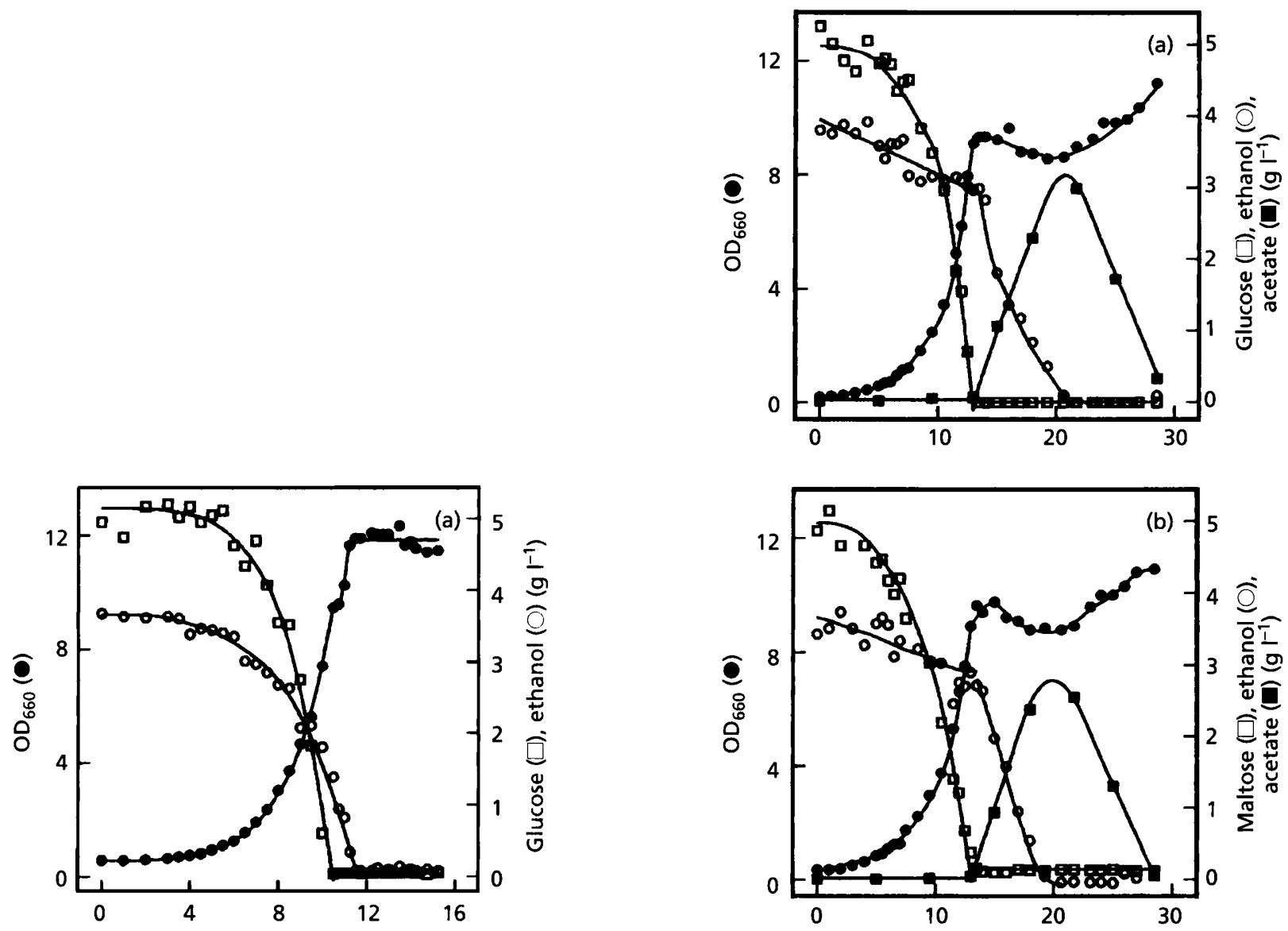

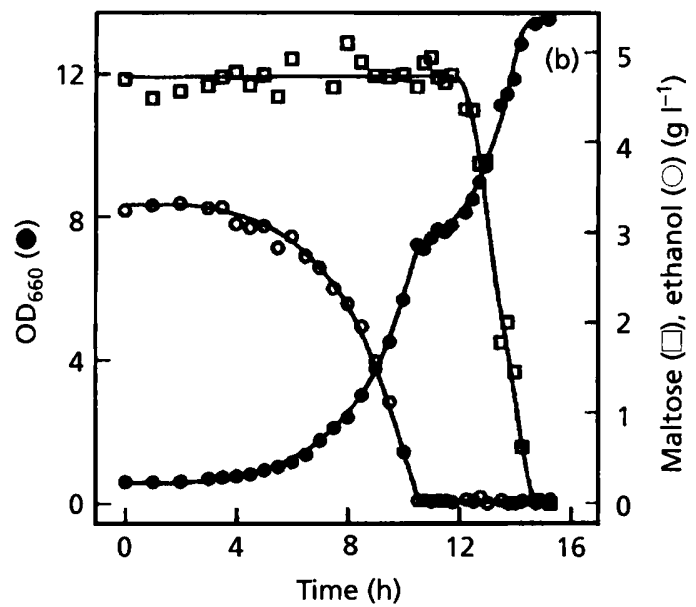

Fig. 4
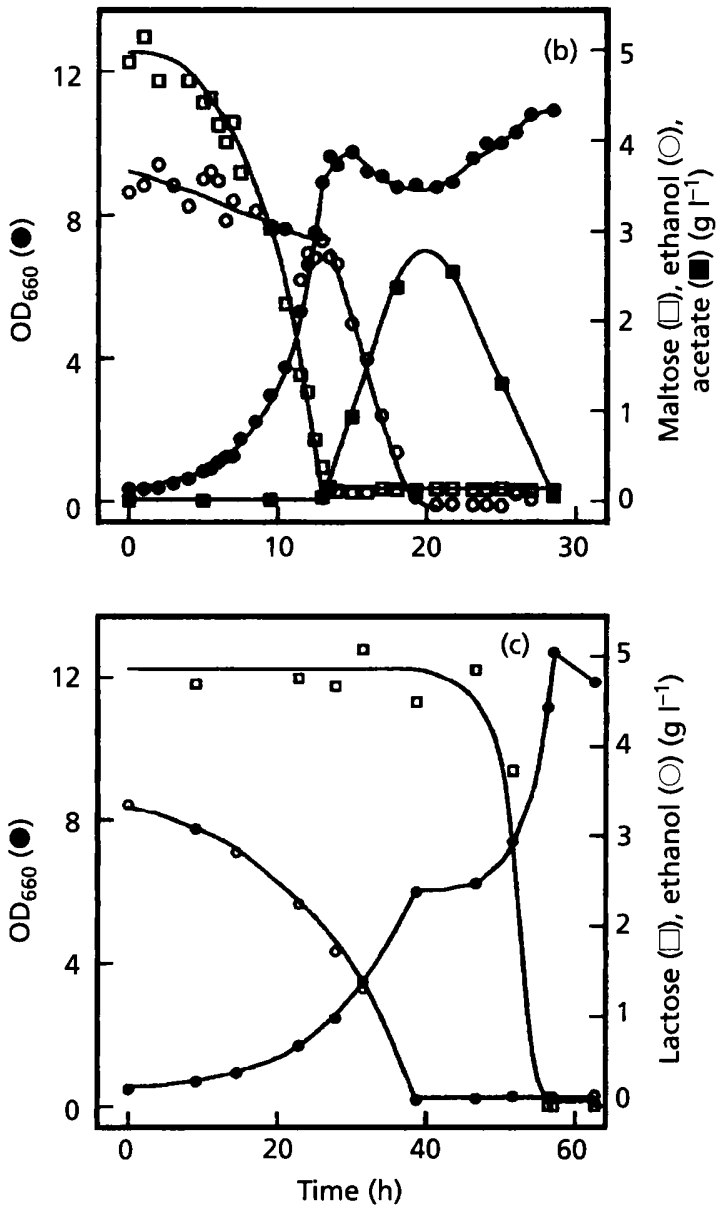

Fig. 5

Fig. 4. Growth and substrate consumption of C. utilis CBS 621 in aerobic, pH-controlled batch cultures on mixtures of (a)

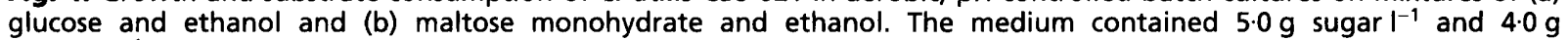
ethanol $\mathrm{I}^{-1}$.

Fig. 5. Growth and substrate consumption of D. castellii CBS 2923 in aerobic, pH-controlled batch cultures on mixtures of (a) glucose and ethanol, (b) maltose monohydrate and ethanol and (c) lactose monohydrate and ethanol. The medium

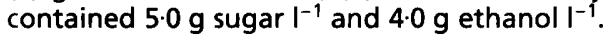

If ethanol, or metabolites directly derived from ethanol, cause the Kluyver effect by inhibition of disaccharide utilization, this should imply that yeasts exhibiting the
Kluyver effect for particular disaccharides preferentially use ethanol when grown on ethanol/disaccharide mixtures. 


\section{Growth of Kluyver-positive yeasts on ethanol/sugar mixtures}

The above hypothesis implies that if $C$. utilis is cultivated batch-wise on a mixture of maltose and ethanol, maltose should be used only after the ethanol has been completely consumed. In contrast, since $C$. utilis is able to ferment glucose, glucose should either be preferred over ethanol or used simultaneously during growth on ethanol/ glucose mixtures.

Batch cultivation of C. utilis was performed in a $\mathrm{pH}-$ controlled, aerobic fermenter with ethanol $\left(4 \mathrm{~g} \mathrm{l}^{-1}\right)$ and maltose $\left(5 \mathrm{~g} \mathrm{l}^{-1}\right)$ as carbon sources. The growth curve showed the classical diauxic pattern (Monod, 1958; Fig. $4 \mathrm{~b})$. During the first phase, growth occurred on ethanol only and maltose was not consumed. After all the ethanol had been consumed, a second growth phase set in, during which maltose was utilized. The two growth phases were separated by a short lag period during which no growth occurred (Fig. 4b).

In a control experiment, $C$. utilis was grown under identical conditions on a mixture of glucose and ethanol (5.0 and $4.0 \mathrm{~g} \mathrm{l}^{-1}$ respectively). In this case, diauxic growth was not observed (Fig. 4a). Glucose and ethanol were utilized simultaneously, until glucose was completely consumed. Growth on ethanol as sole carbon source occurred after a small lag phase, a period possibly needed to induce the enzymes of gluconeogenesis and/or the glyoxylate cycle (Fig. 4a).

To investigate whether preferential utilization of ethanol over disaccharides also occurs in other yeasts exhibiting the Kluyver effect, growth of Debaryomyces castellii CBS 2923 on mixtures of sugars and ethanol was studied. D. castellii is a facultatively fermentative yeast which exhibits the Kluyver effect for lactose, but not for maltose (Sims \& Barnett, 1991).

During growth of $D$. castellii on a mixture of either glucose or maltose and ethanol, diauxic growth was observed (Fig. 5a and Fig. 5b, respectively), with the sugars being preferred over ethanol. After the sugars were completely exhausted, utilization of ethanol proceeded and was accompanied by a transient accumulation of acetate. After this period growth resumed with acetate as carbon source. A diauxic growth pattern was also observed during growth of $D$. castellii on mixtures of lactose and ethanol. However, in this case ethanol was preferred over the sugar (Fig. $5 \mathrm{c}$ ), in accordance with our hypothesis.

\section{DISCUSSION}

The experiments shown in Figs 1 and 2 confirm the conclusion from earlier work that $C$. utilis exhibits the Kluyver effect: this yeast is unable to produce ethanol from maltose, although respiratory metabolism of maltose and fermentation of glucose are both possible. Furthermore, the almost instantaneous occurrence of ethanol formation after the pulse-wise addition of glucose to an oxygen-limited, maltose-grown culture (Fig. 1) confirms our earlier conclusion that $C$. utilis expresses all enzymes required for alcoholic fermentation during oxygen-limited growth on maltose (Weusthuis et al., 1994).

The original aim of this work was to see if the apparent inability of $C$. utilis to produce ethanol from maltose is due to a requirement for extracellular glucose to induce maltose fermentation or that, alternatively, the presence of maltose inhibits glucose fermentation. The results indicate that addition of extracellular glucose does not induce maltose fermentation but, instead, inhibits respiratory maltose metabolism. The observation that this negative effect of glucose addition occurs even under conditions where glucose catabolite repression is not expected (Fig. 2) suggests an alternative explanation for the Kluyver effect, namely that alcoholic fermentation, which occurs after the addition of glucose, inhibits or suppresses maltose metabolism via a feed-back mechanism involving ethanol or a related metabolite (Fig. 3).

The striking correlation, observed in aerobic batchgrowth experiments involving two different yeasts, between the occurrence of the Kluyver effect for a disaccharide and the preferential utilization of ethanol during growth on sugar/ethanol mixtures (Figs 4 and 5) strongly supports the above hypothesis. In fact, the Kluyver effect is an inevitable consequence of the observed preferential use of ethanol over the disaccharides: if, during growth of a micro-organism, a metabolite is preferred as a growth substrate over the compound from which it is formed, net accumulation of the metabolite can by definition not occur.

At present, it is not possible to assess whether regulation is exerted at the level of enzyme synthesis or at the level of enzyme activity or indeed which step in disaccharide metabolism is the target for regulation. Therefore, although the experimental data presented in this paper provide a phenomenological explanation for the occurrence of the Kluyver effect, further work is needed to elucidate the molecular mechanism that causes the apparent down-regulation of disaccharide metabolism in the presence of ethanol. In this respect, it may be necessary to extend studies on the Kluyver effect to yeast species that are well accessible for molecular genetic techniques.

\section{REFERENCES}

Barnett, J. A., Payne, R. W. \& Yarrow, D. (1990). Yeasts: Characteristics and Identification, 2nd edn. Cambridge: Cambridge University Press.

Clifton, D., Walsh, R. B. \& Fraenkel, D. G. (1993). Functional studies of yeast glucokinase. J Bacteriol 175, 3289-3294.

Görts, C. P. M. (1969). Effect of glucose on the activity and the kinetics of the maltose-uptake system and of $\alpha$-glucosidase in Saccharomyces cerevisiae. Biochim Biophys Acta 184, 299-305.

Kluyver, A. J. \& Custers, M. T. J. (1940). The suitability of disaccharides as respiration and assimilation substrates for yeasts which do not ferment these sugars. Antonie Leewwenboek 6, 121-162.

Monod, J. (1958). Recherches sur la Croissance des Cultures Bactériennes. Paris: Herman et Cie.

Peinado, J. M. \& Loureiro-Dias, M. C. (1986). Reversible loss of affinity induced by glucose in the maltose- $\mathrm{H}^{+}$symport of $\mathrm{Sac}$ charomyces cerevisiae. Biochim Biophys Acta 856, 189-192. 
Postma, E., Verduyn, C., Scheffers, W. A. \& van Dijken, J.P. (1989). Enzymic analysis of the Crabtree effect in glucose-limited chemostat cultures of Saccharomyces cerevisiae. Appl Environ Microbiol $55,468-477$

Sims, A. P. \& Barnett, J. A. (1978). The requirement of oxygen for the utilization of maltose, cellobiose and D-galactose by certain anaerobically fermenting yeasts (Kluyver effect). $J$ Gen Microbiol 106, 277- 288.

Sims, A. P. \& Barnett, J. A. (1991). Levels of activity of enzymes involved in anaerobic utilization of sugars by six yeast species: observations towards understanding the Kluyver effect. FEMS Microbiol Lett 77, 295-298.

Sims, A. P., Stålbrand, H. \& Barnett, J. A. (1991). The role of pyruvate decarboxylase in the Kluyver effect in the food yeast, Candida utilis. Yeast 7, 479-487.
Van Leeuwen, C. C. M., Weusthuis, R. A., Postma, E. van den Broek, P. J. A. \& van Dijken, J. P. (1992). Maltose/proton cotransport in Saccharomyces cerevisiae. Comparative study with cells and plasma membrane vesicles. Biochem J 284, 441-445.

Weusthuis, R. A., Adams, H., Scheffers, W. A. \& van Dijken, J. P. (1993). Energetics and kinetics of maltose transport in Saccharomyces cerevisiae: a continuous-culture study. Appl Environ Microbiol 59, 3102-3109.

Weusthuis, R. A., Visser, W., Pronk, J. T., Scheffers, W. A. \& van Dijken, J. P. (1994). Effects of oxygen limitation on sugar metabolism in yeasts: a continuous-culture study of the Kluyver effect. Microbiology 140, 703-715.

Received 29 November 1993; revised 1 February 1994; accepted 9 February 1994. 